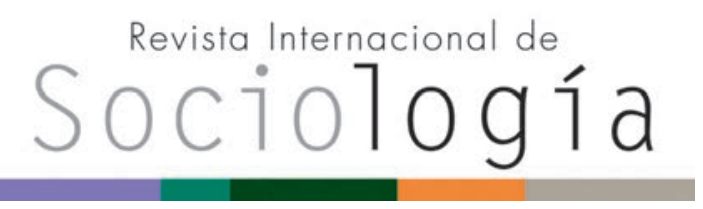

Revista Internacional de Sociología RIS vol. 76 (3), e099, julio-septiembre, 2018, ISSN-L:0034-9712 https://doi.org/10.3989/ris.2018.76.3.17.39

\section{LA FORMACIÓN DE COALICIONES ELECTORALES CONTRANATURALES. Solidaritat Catalana y las elecciones generales de 1907}

\author{
Pedro Lago Peñas \\ Universidad de Santiago de Compostela \\ plpenas@edu.xunta.es \\ ORCID iD: https://orcid.org/0000-0002-1500-6291
}

Cómo citar este artículo / Citation: Lago Peñas, P. 2018. "La formación de coaliciones electorales contranaturales. Solidaritat catalana y las elecciones generales de 1907". Revista Internacional de Sociología 76(3): e099. https://doi.org/10.3989/ris.2018.76.3.17.39

\section{Resumen}

La investigación empírica sobre las preferencias favorables a la formación de coaliciones electorales se ha centrado en partidos con afinidad ideológica. En este artículo argumento que la coordinación electoral también se produce entre partidos ideológicamente distantes con preferencias derivadas afines. Para comprobar estos argumentos recurro al estudio de una coalición electoral entre partidos antagonistas. Analizo la participación de Solidaritat Catalana -integrada por carlistas, regionalistas y republicanos - en las elecciones generales de 1907, por medio de un modelo de coordinación electoral. El análisis empírico recurre a información de la articulación de Solidaritat Catalana; la legislación electoral; las elecciones generales de 1901, 1903, 1905 y 1907, y las elecciones provinciales de marzo de 1907.

\section{Palabras Clave}

Coordinación electoral; Expectativas; Incentivos; Preferencias derivadas; Tasa de descuento.

\section{THE FORMATION OF UNNATURAL ELECTORAL COALITIONS. Solidaritat Catalana and the 1907 national election}

Copyright: (C) 2018 CSIC. Este es un artículo de acceso abierto distribuido bajo los términos de la licencia de uso y distribución Creative Commons Reconocimiento 4.0 Internacional (CC BY 4.0).

Recibido: 13/03/2017. Aceptado: 09/11/2017

\section{Abstract}

Empirical research on preferences about the formation of electoral coalitions is focused on ideologically proximate parties. In this paper I argue that electoral coordination also takes place between ideologically nonproximate parties, but with similar derived preferences. To test this argument I examine the case of an electoral coalition between antagonistic parties, Solidaritat Catalana -formed by carlistas, regionalistas and republicanos - in the 1907 national election. I employ a model of electoral coordination. The empirical analysis relies on information about Solidaritat Catalana, the electoral law, and the results of the 1901, 1903, 1905 and 1907 national elections and the March 1907 province election.

\section{KEYWORDS}

Derived Preferences; Discount Rate; Electoral Coordination; Expectations; Incentives. 


\section{INTRODUCCIÓN}

Para el saber convencional, basado fundamentalmente en la evidencia acumulada sobre alianzas electorales en países democráticos tras la Segunda Guerra Mundial, la probabilidad de que los partidos se coordinen decrece cuanto mayor es su distancia ideológica (Golder 2006). Sin embargo, durante la primera ola de democratización (1828-1926), en Europa la formación de coaliciones electorales entre partidos ideológicamente distantes es un fenómeno habitual. Por ejemplo, en Francia radicales y socialistas se presentan juntos a las elecciones legislativas de 1902. En España Solidaritat Catalana (SC), creada a comienzos de 1906 e integrada por carlistas, regionalistas catalanes y republicanos, concurre a los comicios de 1907 en las circunscripciones catalanas. En Alemania el Partido Popular Progresista (FVP) y el Partido Socialdemócrata Alemán (SPD) forman en 1912 una alianza en la segunda vuelta de las elecciones. Finalmente, en Dinamarca la alianza de radicales y socialistas resulta victoriosa en los comicios de 1913.

¿Cómo se puede explicar, entonces, la coordinación entre partidos antagonistas en las elecciones del primer tercio del siglo XX? El argumento que sostengo en este artículo es que las coaliciones en Francia, España, Alemania y Dinamarca que se apuntan al inicio no son casos anómalos o debidos al azar. La coordinación electoral no es función únicamente de la proximidad ideológica. También puede ocurrir que partidos alejados en el espectro ideológico tengan preferencias derivadas afines. Esto es, partidos que ordenan de la misma forma las alternativas a partir de sus expectativas sobre las consecuencias de las reglas y prácticas institucionales, como el régimen de libertades o las reglas electorales (Benoit 2004; Tsebelis 1990). Estas preferencias derivadas similares posibilitan que partidos ideológicamente distantes pueden alcanzar un acuerdo electoral.

El objetivo empírico de este artículo, más allá de las evidencias anecdóticas que se muestran, es el de comprobar la plausibilidad de la coordinación electoral entre partidos antagonistas como primer paso de una investigación más amplia. Para ello se recurre al estudio de una coalición electoral que pone en cuestión el saber convencional (Gerring 2007). Esto es, se reconstruye la relación causal entre partidos ideológicamente distantes con preferencias derivadas similares y la formación de una alianza electoral. El caso seleccionado es el de SC y su participación en las elecciones generales de 1907. Su interés analítico radica en una de las características que presenta, particularmente exigente para comprobar la posibilidad de coordinación electoral señalada: se alían partidos con una marcada distancia ideológica.

El resto del artículo se divide en cuatro secciones. En la segunda se exponen los argumentos. La ter- cera sección incluye la metodología y los datos utilizados en la investigación. En el apartado siguiente se presenta el análisis empírico de las motivaciones políticas, los incentivos institucionales y políticos y las expectativas de carlistas, regionalistas y republicanos que propician la participación de Solidaridat Catalana en las elecciones de 1907. El quinto apartado recoge las principales conclusiones y se esbozan posibles líneas de investigación.

\section{Argumentos}

La coordinación electoral entre partidos se refiere a un conjunto de procesos mediante los cuales conciertan sus acciones para ganar más escaños o carteras en el gobierno (Cox 2000). Modelada por medio del juego estratégico la batalla de sexos, dicha coordinación se explica por la interacción entre preferencias similares, elevadas tasas de descuento, incentivos institucionales -el sistema electoral-, incentivos políticos - los beneficios netos de la coordinación para cada partido- y expectativas electorales favorables (Blais e Indridason 2007; Golder 2006; Cox 1997). En la literatura las preferencias similares se equiparan a la proximidad ideológica entre los partidos. Frente a ello, en este trabajo se argumenta que, ante la presencia de tasas de descuento, incentivos institucionales, incentivos políticos y expectativas propiciatorios la coordinación electoral también es posible entre partidos antagonistas si tienen preferencias derivadas afines. Dada la naturaleza de la cuestión planteada no se introducen otros elementos analíticos, como la relación causal de las alianzas electorales con la negociación postelectoral o las coaliciones de gobierno (Bandyopahyay, Chatterjee y Sjöstróm 2011; Golder 2006).

Para el saber convencional la sintonía entre partidos es más fácil cuando sus preferencias ideológicas son cercanas. Así, en las democracias actuales de Europa Occidental, es más probable que dos partidos de izquierda compartan políticas que un partido comunista y otro demócrata-cristiano (Golder 2006). Sin embargo, partidos que guardan cierta distancia ideológica pueden converger en una coalición electoral. Basta observar el comportamiento electoral de las organizaciones socialistas durante la primera ola democratizadora para comprobarlo (Reid y Chapman 1995; Luebbert 1991).

En el seno de la II Internacional se plantea la posibilidad de formar alianzas electorales con partidos republicanos o radicales, considerados partidos burgueses. Los defensores de esta estrategia electoral insisten en sus efectos positivos sobre la democratización. Para ellos, la distancia ideológica con estas organizaciones no constituye una barrera infranqueable a la hora de establecer este tipo de acuerdos tácticos. La señalada coalición electoral entre radicales y socialistas daneses propicia la Constitución de 
1915. En Bélgica liberales y socialistas se presentan juntos en 1912 para defender el laicismo, el sufragio universal y un conjunto de reformas sociales.

En términos generales, la competencia electoral en una democracia estable es un escenario más entre varios posibles. Por ello, la formación de preferencias afines puede ir más allá de la cercanía ideológica entre los partidos. Los procesos de democratización o las contraolas antidemocráticas, por citar dos marcos políticos recurrentes, favorecen que partidos alejados en el espacio ideológico tengan preferencias derivadas similares. Esto es, ante la creación o amenaza de destrucción de instituciones políticas, el orden de elección de las diferentes opciones responde a la lógica de un juego estratégico de dos fases. En la primera fase los partidos derivan sus preferencias sobre las alternativas institucionales a partir de sus expectativas sobre los pagos en la segunda fase (Benoit 2004; Tsebelis 1990).

Las reglas y prácticas institucionales sobre asuntos como quiénes pueden votar, las elecciones competitivas o qué libertades y delitos o faltas competen a los tribunales militares constituyen instituciones redistributivas: los beneficios de una parte de la sociedad se producen a expensas de la otra. Como lo normal es que los partidos dispongan de información y conozcan estos conflictos distributivos, deciden en función de los resultados esperados. A causa de ello, resulta factible que partidos alejados en el espectro ideológico tengan preferencias derivadas similares. Un partido republicano y otro socialista pueden estar de acuerdo con el sufragio universal por las consecuencias esperadas de la ampliación del voto, por ejemplo, a los trabajadores industriales. Partidos antagonistas y excluidos pueden coincidir en el rechazo de la manipulación electoral realizada por los partidos gobernantes, para aumentar su número de escaños. O estos mismos partidos pueden querer la libertad de prensa, reunión y manifestación y estar a favor de la desaparición de los tribunales militares que juzgan a detenidos por participar en protestas, para reducir los costes de la movilización ciudadana. En otras palabras, las instituciones redistributivas generan condiciones favorables para que partidos ideológicamente distantes puedan compartir el mismo orden de elección de las diferentes opciones institucionales.

Esta sintonía de preferencias sobre las reglas y prácticas institucionales propicia la coordinación electoral a pesar de las diferencias ideológicas. Los partidos tienen un incentivo para coordinarse y ganar escaños frente a competidores que defienden resultados institucionales contrarios a sus objetivos políticos comunes. Siguiendo con los ejemplos anteriores, se podría tratar del sufragio restringido o la limitación de las libertades democráticas. De lo expuesto se puede extraer la siguiente hipótesis, que completa el modelo de coordinación electoral: cuan- to menor sea la distancia entre las preferencias derivadas de partidos antagonistas, más probable será la alianza electoral.

En definitiva, la coordinación electoral entre partidos ideológicamente distantes constituye una posibilidad que no ha merecido toda la atención requerida en las investigaciones empíricas. La gran mayoría de los datos de que disponen actualmente los especialistas se refieren, casi exclusivamente, a partidos con afinidad ideológica de democracias posteriores a la II Guerra Mundial (Golder 2006). Pero la robustez del modelo de coordinación electoral hay que ponerla a prueba también ante coaliciones de partidos antagonistas.

Por otra parte, las alianzas electorales entre estos partidos también dependen de las tasas de descuento, los incentivos institucionales, los incentivos políticos y las expectativas recogidos en el modelo de coordinación electoral. La tasa de descuento se explica por la racionalidad instrumental de las elites. Caben dos posibilidades, que de nuevo se pueden ilustrar con los debates y decisiones en el marco de la II Internacional. Los partidos socialistas pueden optar por una estrategia a corto plazo y, en consecuencia, darle prioridad a lo que acontece en determinadas elecciones. Por ejemplo, socialistas que se coaligan con republicanos para alcanzar una mayoría parlamentaria. $\mathrm{O}$, por el contrario, deciden apostar por una perspectiva a medio o largo plazo. Es el caso de un partido socialista que espera aumentar sus votos y escaños si se presenta en solitario, y mantiene su programa electoral a lo largo de varias elecciones; o que tiene el temor de perder el apoyo de una parte de su electorado si participa en una alianza electoral con un partido burgués (Cox 1997).

El sistema electoral es una combinación de reglas, fórmulas y mecanismos que traduce los votos emitidos en escaños asignados a candidatos o partidos. Dado que el grado de coordinación de los partidos depende de cuántos votos hacen falta para conseguir los escaños, los incentivos institucionales para coordinarse aumentan cuanto más desproporcional es un sistema electoral ${ }^{1}$. Por tanto, las estrategias de las elites políticas se reducen cuanto más fuerte es un sistema electoral (Lago 2004; Cox 1997; Sartori 1994; Duverger 1987).

La coordinación electoral también depende de que los partidos puedan o no conseguir escaños, o del número de votos que puedan acumular por sí solos. Los distritos electorales reñidos, con acuerdos entre partidos rivales o con equilibrios no duvergerianos ${ }^{2}$, generan incentivos políticos para la coordinación electoral: por esta vía se pueden conseguir más actas de parlamentario. En términos generales, cuanto mayores sean las ganancias de la coordinación para cada partido, más probable será su alianza electoral (Blais e Indridason 2007). 
En la coordinación electoral las expectativas son esenciales. Únicamente cuando las elites políticas disponen de información sobre los posibles efectos de sus acciones, pueden anticipar los resultados y alterar, en su caso, su comportamiento. Es necesario que sepan qué alternativas electorales están en condiciones de obtener escaños y cuáles no, para que la alianza electoral les facilite una ganancia que, de otro modo, no lograrían. Por ello, uno de los desafíos de los acuerdos entre partidos ideológicamente distantes es que sus respectivos partidarios apoyen a candidatos de sus compañeros de alianza. En otras palabras, la certidumbre sobre las posibilidades electorales de los diferentes partidos resulta indispensable para que pueda tener lugar la coordinación (Cox 1997; Lago 2004).

En definitiva, las cinco hipótesis para la investigación empírica de la coordinación electoral entre partidos antagonistas, que funcionan interactivamente bajo la condición ceteris paribus, son las siguientes:

- $\mathrm{H}_{1}$ : Cuanto menor sea la distancia entre las preferencias derivadas de los partidos, más probable será la alianza electoral.

- $\quad \mathrm{H}_{2}$ : Cuanto más altas sean las tasas de descuento de los partidos, más probable será la alianza electoral.

- $\mathrm{H}_{3}$ : Cuanto más desproporcional sea un sistema electoral, más probable será la alianza electoral.

- $\mathrm{H}_{4}$ : Cuanto mayor sea el beneficio neto de la cooperación para cada partido, más probable será la alianza electoral

- $\mathrm{H}_{5}$ : Cuanto más favorables sean las expectativas electorales, más probable será la alianza electoral.

\section{MetOdología Y DATOS}

La investigación explica por qué carlistas, regionalistas y republicanos se coaligan y pueden acumular más escaños que concurriendo en solitario en las elecciones generales de abril de 1907, mediante el modelo de coordinación electoral presentado en el apartado anterior. La decisión de coaligarse depende de las preferencias derivadas, las tasas de descuento, los incentivos institucionales, los incentivos políticos y la información sobre las posibilidades electorales de SC. Carlistas, regionalistas y republicanos poseen preferencias derivadas similares y elevadas tasas de descuento. El sistema electoral de la Restauración es mayoritario. El Partido Liberal (PL) y el Partido Conservador (PC), incapaces de manipular los resultados electorales como en comicios anteriores, se coordinan en todos los distritos uninominales donde se presenta SC, su rival más fuerte. Con las cercanas elecciones generales en perspectiva y los resultados de las elecciones provinciales de marzo de 1907 en mente, los dirigentes carlistas, regionalistas y republicanos saben que SC puede incrementar el número de escaños de los partidos coaligados gracias a la concentración de voto.

El estudio de las preferencias derivadas, las tasas de descuento, los incentivos institucionales, los incentivos políticos y las expectativas electorales combina el análisis cualitativo y cuantitativo, y se realiza a partir de información proveniente de la minuciosa investigación realizada por historiadores y politólogos de SC y de la movilización política y electoral en Cataluña desde finales del siglo XIX. Sin esta intensiva investigación sobre qué ocurre no se podría responder a la pregunta planteada en este trabajo: por qué ocurre.

Las preferencias derivadas y las tasas de descuento propiciatorias se observan al comparar las estrategias electorales —reconstruidas por los investigadores a través de cartas, órganos de prensa o manifiestos electorales-de carlistas, regionalistas y republicanos antes de SC y durante el proceso de formación de la coalición electoral. Los incentivos institucionales vienen dados, sobre todo, por la Ley Electoral de 1878, que establece las reglas, fórmulas y mecanismos que traducen los votos emitidos por los electores en escaños. Los incentivos políticos se conocen a través del análisis de las huellas dejadas por la movilización de los partidos opositores - que se traduce en la autentificación del sufragio y en el aumento de sus votos y escaños - en las elecciones generales de 1901, 1903 y 1905 y las elecciones provinciales de marzo de 1907; la actitud de las autoridades en vísperas de las elecciones generales de 1907, y la coordinación electoral de liberales y conservadores en 32 de los 34 distritos uninominales de Cataluña en los comicios de 1907, aunque acaban retirándose en quince de ellos. La información sobre las expectativas procede de los resultados alcanzados por SC en las elecciones provinciales de marzo de 1907.

Los resultados electorales de las elecciones generales de abril de 1907 se recogen en el anexo. Hay 34 circunscripciones uninominales - se elige un único escaño-y dos multinominales - Barcelona, con siete escaños y Tarragona-Reus-Falset, con tres escaños- en Cataluña. SC obtiene cuarenta de los 44 escaños en disputa (91 por ciento); de los cuatro restantes, tres son para el PC (7 por ciento) y uno para el PL (2 por ciento). En los comicios de 1905 liberales y conservadores ganan veintiséis escaños (59 por ciento); los republicanos, once (25 por ciento) y los regionalistas, siete (16 por ciento). En 190 SC gana en treinta de los 32 distritos uninominales donde se presenta. Asimismo, esta coalición electoral resulta victoriosa en los dos distritos multinominales. Como en estos distritos el voto es limitado hay una proporción entre los votos de cada votante y los escaños del distrito; 5/7 en Barcelona y 2/3 en Tarragona-Reus-Falset-, SC adopta la estrategia de copar todos los escaños. Sus dirigentes diseñan un plan para la distribución de votos y electores. 


\section{ANÁLISIS EMPÍRICO}

El carlismo constituye un partido monárquico de señalado carácter antiliberal, surgido en las postrimerías del Antiguo Régimen. Los integrantes de la Lliga Regionalista (LIR), partido fundado en 1901, son nacionalistas catalanes conservadores. El republicanismo se caracteriza por su heterogeneidad ideológica y organizativa. Coexisten los republicanos federales, los republicanos nacionalistas y la Unión Republicana (UR); si bien este último partido, creado en 1903 y dirigido por Nicolás Salmerón, tampoco es monolítico.

Para el objetivo de este estudio, resulta especialmente interesante el sector de la UR encabezado por Alejandro Lerroux, opuesto a la formación de SC y que tiene en Barcelona su núcleo organizativo y electoral. Las posiciones enfrentadas de Salmerón y Lerroux dentro de la UR, a causa de la participación en SC, acaban generando una fractura organizativa y electoral en el partido. En marzo de 1906 se le retira a Lerroux la dirección de La Publicidad, órgano de prensa de la UR de Barcelona. A la concentración multitudinaria de SC en Barcelona el 20 de mayo de 1906, Lerroux responde con una reunión de la Junta Municipal de Barcelona de la UR, que declara inmoral la alianza con fuerzas monárquicas. En junio de 1906 Lerroux recupera un órgano de prensa en Barcelona, con la fundación de El Progreso, al que más tarde se suman El Descamisado y La Rebeldía. En el otoño de 1906 los republicanos antisolidarios le envían a Salmerón una carta con 15.000 firmas pidiendo la salida de la UR de SC. Durante estos meses se suceden agresiones físicas. En diciembre de 1906 se materializa la ruptura en la UR de Barcelona, con la formación de dos juntas municipales. Finalmente, los republicanos antisolidarios de Lerroux presentan candidaturas a las elecciones provinciales de marzo y las generales de abril de 1907, frente a las de SC (Álvarez Junco 1990; Romero 1989; Culla 1986; Artola 1974).

En el carlismo se inicia, desde finales del siglo XIX, un proceso de reorganización y reorientación electoral. Con respecto a lo primero, los círculos tradicionalistas se convierten en los núcleos de estructuración de su base social. Así, los carlistas disponen en 1896 de cien círculos en Cataluña. Dotados de un local, cumplen tres funciones: política - acogen a las juntas tradicionalistas y son centros de propaganda-, de formación e instrucción y de cohesión y recreo. En cuanto a la reorientación electoral, el final del retraimiento electoral posibilita a este partido la difusión de sus ideas y el acceso a las Cortes, las diputaciones provinciales y los ayuntamientos (Canal 1998; 2006).

La propia formación de la LIR está vinculada a unas elecciones generales. El Centre Nacional Català y la Unió Regionalista se fusionan y presentan la "candidatura de los cuatro presidentes" a los comicios de mayo de 1901 (Molas 1972: cap. 3). Como Cambó escribe en un artículo publicado unas semanas antes en La Veu de Catalunya -20-III1901- (Riquer 1977: 192-193):

"Catalunya no té més que dos camins a seguir: la lluita electoral o la revolució. Mes com aquesta no la creiem avui posible, ni encara que ho fos, la creuríem convenient, aquests dos camins se redueixen a un de sol: a procurar que al Parlament hi vagin homes que hi portin la seva veu, el seu esperit, les seves reclamacions, fins si convé les seves amenaces".

La UR, con Lerroux de protagonista, se convierte en un partido de masas en Barcelona. Pasa a disponer de un amplio tejido asociativo en los barrios de la ciudad y organizaciones sectoriales propias. Este entramado organizativo cumple múltiples funciones: políticas, asistenciales, educativas y culturales. Lerroux promueve en Barcelona lo que observa en Bruselas durante su viaje de 1902. Esta movilización política se traslada al campo electoral, en oposición a los liberales, conservadores y regionalistas (Álvarez Junco 1990; Romero 1989; Culla 1986).

\section{A. Preferencias derivadas $Y$ TASAS DE DESCUENTO}

Desde 1901 y hasta de la formación de SC, regionalistas y republicanos son rivales políticos en las diferentes citas electorales. $Y$ en la ciudad de Barcelona la LIR y la UR se convierten en las fuerzas contrincantes por excelencia, ante la debilidad de liberales y conservadores. Sus preferencias ideológicas divergentes y su enfrentamiento político que se extiende a otros escenarios, como la huelga general de 1902- hacen inviable que puedan converger en una alianza electoral frente al PL y el PC. Por otra parte, los regionalistas sí están abiertos a la coordinación electoral con otras formaciones políticas conservadoras, como los carlistas (Balcells, Culla y Mir 1982; Molas 1972).

Entre las iniciativas de aproximación se encuentra la propuesta de los carlistas de entrar en negociaciones con los regionalistas tras los buenos resultados alcanzados por la LIR en las elecciones generales de 1901. Joan Bardina, líder carlista, le hace este ofrecimiento a Prat de la Riba, dirigente de la LIR, en una carta sin fechar -aunque se cree que es de mayo o junio de 1901-(Riquer 1977: 341):

"Li escrich la present per encarrech d'una Comissió, no per pura voluntat meva. Es el cas que d'una reunió de personalitats carlinas ne sortí, fá pocs dies, I'idea d'orientar el Carlisme cap al Catalanisme, y, com a consecuencia, que's devía demanar a don Jaume permís pera entrar en negociacions ab els catalanistas, si aquests s'hi prestaven".

La relación entre la LIR y la UR da un giro brusco a finales de 1905. Tras las elecciones municipales del 12 de noviembre, beneficiosas para la LIR en Barcelona y otros municipios de Cataluña, una viñeta publicada por el semanario catalanista $\mathrm{Cu}$-Cut gene- 
ra rechazo en el ejército. En la noche del 25 al 26 de noviembre unos trescientos militares de la guarnición de Barcelona, uniformados y armados, asaltan y saquean su sede y la de La Veu de Catalunya, otra publicación regionalista. Este incidente y sus consecuencias monopolizan la vida política española durante meses. Los militares, además de considerarse exentos de toda culpa, reclaman castigo inmediato y medidas legales para quienes atenten contra la unidad nacional o el honor de las fuerzas armadas. El gobierno promueve la suspensión de las garantías constitucionales en Barcelona para controlar la situación. Durante el debate parlamentario en el Congreso de los Diputados, Salmerón propone de forma inesperada a la LIR unirse para oponerse a la citada medida y hacer frente a las acusaciones de independentismo dirigidas a los regionalistas catalanes. Estos se muestran de acuerdo. Los diputados de ambos partidos votan contra la supresión de las garantías constitucionales y se oponen a la Ley de Jurisdicciones, que pretende regular los delitos de prensa, elaborada por el gobierno liberal de Moret.

Los contactos establecidos con otros partidos implantados en Cataluña llevan a la formación de SC a principios de 1906. Además de la UR y la LIR, participan los carlistas, los republicanos nacionalistas y los republicanos federales. En sus primeros meses de existencia, la actividad de SC se concentra en dos frentes. En el Congreso de los Diputados, los parlamentarios carlistas, regionalistas y republicanos rechazan la Ley de Jurisdicciones, que se aprueba el 23 de marzo de 1906. En la calle, lanzan una campaña de movilización política. Entre los numerosos actos públicos organizados, sobresalen el mitin del 11 de febrero en Girona y la concentración del 20 de mayo en Barcelona, con la participación de decenas de miles de personas. Estimulados por la movilización ciudadana, los regionalistas le plantean a los restantes partidos convertir SC en una alianza electoral y enfrentarse conjuntamente a la manipulación electoral, alianza que se estrena en las elecciones provinciales de marzo de 1907.

Para Salmerón la alianza electoral de la UR con carlistas y regionalistas supone una oportunidad para ampliar el frente anticaciquil, purificar el sufragio y democratizar España. En sus manifestaciones públicas insiste, sobre todo, en cuatro argumentos. En primer lugar, el sistema político de la Restauración ha provocado un divorcio entre el Estado y la nación. SC posibilita incorporar a otras fuerzas a la tarea de regeneración política. Se trata del primer acto de emancipación nacional, pero no de Cataluña como nación, sino de una región española que inicia la liberación de las garras de un sistema corrupto. En segundo lugar, el bloque anticaciquil se puede ampliar a todas las formaciones políticas ajenas a las redes clientelares del PL y el PC dispuestas a enfrentarse a la corrupción política. En tercer lugar, la entente solidaria no debe circunscribirse a Cataluña. Debe extenderse al resto de España y, de forma especial, a las regiones históricas, donde las demandas de autonomía política pueden ser canalizadas en contra del sistema de la Restauración. En cuarto lugar, la autentificación del sufragio y el final del caciquismo se relacionan con el cambio de régimen. Del beneficio electoral esperado Salmerón deriva la idea de que la superación de la ruptura entre el Estado y la nación conlleva la afirmación republicana (Martínez 2006a; 2006b; 2008a; 2008b; Duarte 2004; Cortina 1992; Álvarez Junco 1990).

De acuerdo con las estrategias electorales de las elites carlistas, regionalistas y republicanas antes y después de la formación de SC, se pueden establecer las siguientes relaciones entre las preferencias ideológicas y derivadas, las tasas de descuento expuestas y la coordinación electoral. La distancia ideológica entre los republicanos y los regionalistas y carlistas imposibilita su confluencia en una coalición electoral: eligen presentarse separados frente a la coordinación con partidos alejados en el espectro político para ganar más escaños. En cambio, su posición común sobre determinadas reglas y prácticas institucionales posibilita su alianza electoral: eligen presentarse juntos a las elecciones y aumentar sus escaños frente a su concurrencia en solitario. Mientras sus preferencias ideológicas antagónicas les llevan a la descoordinación, sus preferencias derivadas afines les conducen a la coordinación.

En resumen, las motivaciones políticas de carlistas, regionalistas y republicanos para convertir SC en una coalición electoral confirman las dos primeras hipótesis. Estas formaciones políticas, a pesar de sus divergencias ideológicas, comparten preferencias derivadas sobre determinadas reglas y prácticas institucionales. Están en contra de la Ley de Jurisdicciones y rechazan el fraude electoral realizado por los partidos gobernantes. Demandas que se complementan, en el programa electoral de las elecciones generales de 1907, con la reivindicación de la descentralización administrativa. Asimismo, estas elites políticas tienen altas tasas de descuento. Prefieren trasladar la exitosa movilización conjunta contra la Ley de Jurisdicciones a las inmediatas elecciones, por medio de una coalición para ganar más escaños, que presentarse en solitario con su programa electoral.

\section{B. INCENTIVOS INSTITUCIONALES}

La Ley Electoral de 1878 —completada con la Ley Electoral de 1890, que introduce el sufragio masculino universal- incluye las reglas, fórmulas y mecanismos que traducen en escaños los votos emitidos por los electores a partidos o candidatos. El sistema electoral adopta la regla de la mayoría relativa —el ganador es la alternativa con mayor número de votos, sin necesidad de alcanzar una mayoría u otra 
cuota cualquiera - en una combinación de distritos uninominales con voto único y distritos multinominales con voto limitado, inspirado en el modelo de Gran Bretaña entre 1867 y 1885. Las proporciones entre los votos de cada votante y los escaños del distrito son $2 / 3,3 / 4,3 / 5,5 / 7$ y 6/8. Hay 307 distritos uninominales -34 de ellos en Cataluña-, la mayoría en zonas rurales; y veintiséis distritos multinominales —dos en Cataluña: Barcelona, con siete escaños y Tarragona-Reus-Falset, con tres escaños-, con magnitudes entre tres y ocho escaños, que suponen un total de 90 escaños, en zonas urbanas. Los escaños de cada distrito multinominal se asignan a la candidatura más votada, que recibe el número de escaños por los que cada votante puede votar -alrededor de dos tercios- y a la siguiente candidatura en votos, que recibe el escaño o los escaños restantes. En síntesis, alrededor de un 75 por ciento de los escaños son elegidos en distritos uninominales -77 por ciento en Cataluña-, mientras que hasta un 93 por ciento son asignados a las candidaturas con una mayoría relativa de votos en cada circunscripción —93 por ciento en Cataluña- (Colomer 2004).

Este escenario institucional propiciatorio de la coordinación electoral -como se recoge en la tercera hipótesis - no pasa inadvertido para las elites regionalistas, impulsoras de la conversión de SC en una alianza electoral. Pabón (1952: 215) narra en su biografía de Cambó —líder de la LIR — el siguiente episodio, cuyo origen se encuentra en un trabajo previo de Pla:

"Desde la frontera, Cambó se había dirigido a Toulouse, donde encontró a Puig y Cadafalch y a Ventura, emigrados también. Observaron desde la ciudad francesa el curso de los acontecimientos. Puig y Ventosa marcharon a Paris y Cambó volvió a Barcelona. Fernando Agulló, que se encontraba casualmente en el andén de la estación de Caldas de Malavella al paso del tren, subió en él para acompañarle y le preguntó:

—¿Qué ha de hacerse ahora?

—Se ha de hacer eso — -le respondió Cambó, alargándole un papel.

Era un mapa donde se hallaban gráficamente distribuidas las fuerzas políticas y donde cada distrito, en relación con dichas fuerzas, tenía asignado el nombre de un representante. Plan que, año y medio después, con pocas variantes, sería aplicado en las elecciones de la Solidaridad".

Los resultados alcanzados por SC en las elecciones generales de 1907 muestran el beneficio de la coordinación electoral. Esta alianza electoral gana en treinta de las 32 circunscripciones uninominales donde se presenta, con un porcentaje medio del 78,27 por ciento de los votos. La pregunta clave, por supuesto, es en qué medida la coalición electoral resulta decisiva para conseguir los escaños en juego, esto es: si los partidos en solitario habrían sido capaces de ganar. De acuerdo con los resultados de las elecciones inmediatamente anteriores -las de 1905, en las que no hay una alianza electoral-, la media del porcentaje de voto del partido con más apoyo en cada distrito de los que forman la coalición en 1907 es del 34,43 por ciento, y solo logran nueve escaños (Balcells, Culla y Mir 1982: 509-518).

\section{INCENTIVOS POLÍtICOS}

El PL y el PC dominan las circunscripciones catalanas hasta principios del siglo XX, tanto antes como después de la aprobación del sufragio universal masculino en 1890. Liberales y conservadores recurren a una panoplia de cursos de acción, susceptibles de diferentes combinaciones, para alcanzar la victoria electoral. Entre ellos destacan los cinco siguientes. Primero, se alcanza un acuerdo entre los partidos del distrito electoral y las autoridades gubernamentales. Segundo, se activa el fraude electoral por medio de la constitución irregular e ilegal de las mesas electorales, el despido de los interventores de los partidos opositores, el arreglo de la lista de votantes y la firma de actas en blanco, que son enviadas al candidato adicto para que dé su conformidad y las remita a la Junta de Escrutinio. Tercero, se hace uso del intercambio de votos por favores, ya sea por medio de la compra monetaria del voto, la tramitación de expedientes ante la administración, la liberación de la obligación del cumplimiento del servicio militar o el apoyo en subastas de aprovechamientos de pastos. Cuarto, se utiliza la fuerza física contra los opositores. Quinto, en circunscripciones electorales disputadas, liberales y conservadores se coordinan y presentan un único candidato (Rubí y Armengol 2001; Balcells, Culla y Mir 1982).

Se trata de una estrategia electoral contra la que están prevenidos carlistas, regionalistas y republicanos. Por ejemplo, en los comicios de 1891 los distritos gerundenses se ven agitados por la presentación de candidaturas republicanas y carlistas. Los resultados muestran al PL y al PC, y también a los partidos de oposición, las ventajas de la coordinación electoral. En las elecciones de 1893 los republicanos se presentan en cinco de los ocho distritos, uno más que dos años antes, pero liberales y conservadores recuperan el acta de Figueres al presentar como único candidato al liberal Baró. La alianza electoral entre el PL y el PC se amplía en las elecciones de 1903 y 1905, cuando aumenta la competencia electoral en una parte de las circunscripciones uninominales. Se trata de no dividir el voto en distritos reñidos (Rubí y Armengol 2001: 269; Balcells, Culla y Mir 1982: 376).

Pero el panorama político cambia en el distrito de Barcelona desde las elecciones generales de 1901 y en otros desde las de 1903 y 1905. Se propaga la autentificación del sufragio y los partidos opositores ganan votos y escaños frente a liberales y conserva- 
dores. Un giro propiciado por la movilización política y electoral de carlistas, regionalistas y republicanos (Rubí y Armengol 2012; Balcells, Culla y Mir 1982).

En Barcelona los comicios del día 19 de mayo de 1901 suponen el final del dominio del PL y el PC y la LIR irrumpe como fuerza antagonista junto a los republicanos. Cada partido opositor organiza por su cuenta, pero en un clima de colaboración frente a liberales y conservadores, un sistema de control de las urnas y del escrutinio general de actas que impide el inflado de los votos de las candidaturas liberal y conservadora. Regionalistas y republicanos establecen un amplio sistema de vigilancia de la votación. La LIR dispone de interventores en las 213 mesas electorales y oficinas electorales en todos los distritos de la ciudad. A las cuatro de la tarde cierran los colegios electorales y comienza el recuento de votos en presencia de interventores de la oposición. Los pertenecientes a la LIR permanecen hasta el final y consiguen una copia certificada de los resultados de sus respectivas secciones. Pero el Gobierno Civil falsea las actas antes de su llegada a la Junta de Escrutinio. Lerroux convoca el día 23 a sus seguidores frente a la Diputación Provincial, donde la Junta de Escrutinio procede al recuento. Los funcionarios recurren a los datos de los interventores regionalistas para la verificación de los resultados de cada una de las 213 secciones electorales de la ciudad. Se proclaman diputados por Barcelona a cuatro candidatos regionalistas, dos republicanos y uno monárquico (Rubí y Armengol 2001; Álvarez Junco 1990; Romero 1989; Riquer 1977; Molas 1972).

En una parte de las restantes circunscripciones de Cataluña se observan tres fenómenos en las elecciones generales de 1903 y 1905. En primer lugar, los partidos opositores incrementan su persecución del fraude electoral. Se comprueba, por ejemplo, en las actas impugnadas por irregularidades o fraude: las de cuatro circunscripciones en 1901, nueve en 1903 y ocho en 1905. En segundo lugar, estos partidos presentan más candidatos. El número de distritos con candidato único pasa de catorce en 1901 a cuatro en 1903 y 1905 . En tercer lugar, como se observa en el cuadro 1, aumenta el número de diputados carlistas, regionalistas y republicanos: once en 1901, diecinueve en 1903 y dieciocho en 1905, de un total de 44 (Rubí y Armengol 2001; Balcells, Culla y Mir 1982: cap. 13).
En las elecciones provinciales de marzo de 1907 se logra la limpieza electoral en los distritos donde compite SC. Y en los comicios de abril se generaliza la autentificación del sufragio. La movilización electoral de carlistas, regionalistas y republicanos resulta determinante (Rubí y Armengol 2012; Balcells, Culla y Mir 1982). El pesimismo de las autoridades es manifiesto en su correspondencia interna desde semanas antes de las elecciones generales. Osorio y Gallardo, Gobernador Civil de Barcelona, le comunica por carta (7-III-1907) a La Cierva, Ministro de Gobernación, lo siguiente (Pabón 1952: 220):

\begin{abstract}
"No hay que pensar en nada. Nuestros mismos correligionarios lo reconocen (...) Todo lo que se intente contra el catalanismo antes de las elecciones será inútil y sólo contribuirá a estrechar por necesidades de defensa los vínculos solidarios y a privarnos de paz en el presente y de autoridad en el porvenir".
\end{abstract}

Y La Cierva le transmite (carta del 10-IV-1907) a Maura, Presidente del Gobierno, un mensaje parecido (Pabón 1952: 221):

"Como verá, lo de la Solidaridad constituye una verdadera borrachera. Políticamente, ya no se puede andar por la calle sin titularse solidario ... Triste es nuestra situación ...".

Como concluye Cuadrado (1969: 748), ante la expectativa del fracaso electoral de liberales y conservadores en los distritos electorales de Cataluña, "desde una semana antes de la fecha electoral (en el nombramiento y proclamación de interventores que se hacía el domingo anterior), se acentúa la presión ministerial en el resto de provincias".

En este nuevo escenario de elecciones competitivas, el PL y el PC se alían en todos los distritos uninominales reñidos, como se observa en el anexo y en el cuadro 2. En 32 de las 34 circunscripciones deciden presentar un único candidato, liberal o conservador, para no dividir el voto frente a SC. En diecisiete de ellas los aspirantes se mantienen hasta el final (Balcells, Culla y Mir 1982: 123). En las quince restantes, en previsión de un resultado adverso, los candidatos, "presentidos y hasta anunciados", no concurren (Romero 1989: 399). En los otros dos distritos (Sort y Roquetes), donde SC no participa y los seguidores de Lerroux o son débiles o no se presentan, compiten candidatos de los dos partidos. La literatura explica la retirada de libera-

Cuadro 1.

Distribución de los escaños de los distritos de Cataluña

\begin{tabular}{|l|c|c|c|}
\hline & 1901 & 1903 & 1905 \\
\hline PL y PC & 33 & 25 & 26 \\
\hline Carlistas & 1 & - & - \\
\hline Regionalistas & 6 & 5 & 7 \\
\hline Republicanos & 4 & 14 & 11 \\
\hline
\end{tabular}

Fuente: elaboración propia a partir de Balcells, Culla y Mir 1982: 375. 
les y conservadores como un efecto de la movilización electoral conjunta de carlistas, regionalistas y republicanos (Rubí y Armengol 2012: cap. 6; Balcells, Culla y Mir 1982: 376). Los resultados electorales apoyan esta interpretación. En 1907, en los quince distritos en los que no se presentan liberales y conservadores, hay una participación del 53,01 por ciento y en 1905 - liberales y conservadores no concurren en dos de estas quince circunscripciones_la participación es de 52,77 por ciento (Balcells, Culla y Mir 1982: 508-529). La diferencia no es estadísticamente significativa.

En cuanto a las circunscripciones multinominales, el PL y el PC en Barcelona no concurren pero sí los candidatos partidarios de Lerroux-, y en Tarragona-Reus-Falset cada partido presenta un candidato. SC adopta - como en las elecciones provinciales de marzo de 1907 en el segundo distrito de Barcelona - la estrategia de copar todos los escaños (Riquer 1972). La distribución proyectada de votos y electores en los distritos de esta ciudad se recoge en el anexo.

En resumen, los movimientos estratégicos del PL, el PC y SC confirman la cuarta hipótesis: cuanto mayor sea el beneficio neto de la cooperación para cada partido, más probable será la alianza electoral EI acuerdo electoral entre liberales y conservadores en las circunscripciones reñidas favorece la coordinación electoral entre carlistas, regionalistas y republicanos.

\section{EXPECTATIVAS ELECTORALES}

Por lo que respecta a las expectativas, las elecciones provinciales, consideradas tradicionalmente de orden secundario, se convierten en un test. Convocadas por el gobierno conservador de Maura para el 10 de marzo de 1907, un año después de la formación de SC y poco antes de las elecciones gene- rales, sus resultados facilitan a las elites carlistas, regionalistas y republicanas información sobre las posibilidades de $\mathrm{SC}^{3}$. Afecta a once distritos de Cataluña. SC presenta candidaturas oficiales en seis (cuatro de la provincia de Barcelona y dos de la de Girona) y compite con los republicanos antisolidarios de Lerroux en dos de Barcelona ciudad; y con el PL y el PC en tres (Vilanova-Sant Feliu, Girona y Olot-Puigcerdà) (Álvarez Junco 1990: 328; Camps 1970: 70).

Lerroux rechaza públicamente la participación en SC desde el inicio, antes de esta primera experiencia electoral. Argumenta que las coincidencias entre la UR y las demás formaciones políticas, en particular carlistas y regionalistas, son menores que las diferencias. Se comprueba en el análisis realizado por Romero (1989: 358, 367-368) de dos artículos del líder republicano: El alma en los labios (La Publicidad, 9-XII-1905) y Mi Evangelio (La Campana de Gràcia, 7-IV-1906). En ambos escritos se observa su disconformidad con la posición de Salmerón. En el primer documento, Lerroux reivindica la lucha política de la UR contra los regionalistas en la ciudad de Barcelona: "Hace menos de seis años imperaba en Barcelona el catalanismo político, hijo degenerado de un contubernio monstruoso (...) Con tal estado de cosas acabó el partido republicano". Se pregunta por qué, cuando en anteriores ocasiones republicanos y liberales sufrieron ataques, "no se viola el derecho y cuando se ataca a los separatistas sí". Recuerda que en la huelga general de Barcelona de 1902 "la canalla separatista agasajaba a los oficiales y obsequiaba a los soldados, que entonces salían a defender, no el orden, por nadie alterado, sino la propiedad de los separatistas y entonces no ardió nada, pero corrió la sangre del pueblo". Afirma que, si llega a encontrarse en Barcelona el 25 de noviembre de 1905, habría emulado a los militares y que cooperar con la LIG supone renunciar al

\section{Cuadro 2.}

Elecciones generales de abril de 1907

Candidatos del PL y el PC en los distritos uninominales de Cataluña

\begin{tabular}{|c|c|c|}
\hline Distritos con candidato único & Distritos con candidato único que no concurre & Distritos con dos candidatos \\
\hline Arenys & Castellterçol & Sort \\
Balager & Cervera \\
Berga & Figueres & \\
Gandesa & Girona & \\
Igualada & Granollers & \\
La Seu & La Bisbal & \\
Les Borges & Mataró & \\
Lleida & Olot & \\
Manresa & Sabadell & \\
Puigcerdà & Sant Feliu & \\
Sta. Coloma & Terrasa & \\
Solsona & Vic & \\
Torroella & Vilademuls & \\
Tortosa & Vilafranca & \\
Tremp & Vilanova & \\
Valls & & \\
Vendrell & & \\
\hline
\end{tabular}

Fuente: elaboración propia a partir de Balcells, Culla y Mir (1982: 520-529). 
deseado respaldo del ejército. Finaliza el artículo con estas palabras: "antes que pactar con esa chusma envilecida por el amor al ochavo, que es la quintaesencia de su regionalismo separatista, estoy dispuesto a rebelarme contra el mundo, acompañado o solo".

En el segundo documento, el dirigente republicano aduce que SC implica el reconocimiento de la prioridad del autonomismo regional sobre otras soluciones políticas, cuando los republicanos han antepuesto siempre la autonomía individual a la municipal y la local a la regional. Considera la alianza electoral de los republicanos con los carlistas y regionalistas catalanes una unión de elementos políticos antitéticos (obreros y patronos; ateos, escépticos y creyentes; demócratas y absolutistas), sin haber urgencia alguna que justifique la iniciativa; tampoco es el resultado de acuerdos políticos que reflejen un cambio significativo en las aspiraciones de los grupos no republicanos. Menciona episodios recientes de la conflictividad laboral en Cataluña y la competencia electoral marcados por la hostilidad entre la UR y la LIR. Rememora también que en elecciones anteriores la UR se había negado a pactar, no ya con la LIR, sino hasta con los republicanos federales. Por último, niega que SC pueda lograr la abrogación de la Ley de Jurisdicciones, que ofrezca nada útil a los obreros y republicanos y que alianzas electrales como SC sirvan para algo en aquella coyuntura política.

Como se puede observar en el cuadro 3, SC logra un excelente resultado electoral. Consigue diecinue- ve representantes, mientras que los liberales obtienen dos y los conservadores y republicanos antisolidarios de Lerroux se quedan en uno. Con ello las elites carlistas, regionalistas y republicanas logran superar uno de los desafíos de los acuerdos entre partidos ideológicamente distantes. De hecho, en las elecciones generales de abril de 1907 surgen tensiones por este motivo. Dos ejemplos: la proclamación de candidatos en las circunscripciones de Sant Feliu, Vilanova y Granollers se produce en un clima de división; en Tarragona, como recoge La Cruz (18-IV-1907), elementos católicos no quieren votar a un candidato por ser "republicano y anticatólico" (Balcells, Culla y Mir 1982: cap. 5).

La autentificación del sufragio y los resultados electorales positivos animan a SC a concurrir a las elecciones generales de abril de 1907. Una semana antes de los comicios, los partidos coaligados rubrican en un mitin multitudinario su alianza electoral y presentan el Programa de Tívoli. Demandan la derogación de la Ley de Jurisdicciones, la dignificación del sufragio y amplia autonomía administrativa para regiones y municipios. En conclusión, se trata de un resultado que respalda la quinta hipótesis: las expectativas electorales favorables posibilitan la coordinación electoral.

No obstante, como figura en el cuadro 4, la cuantía de los votos alcanzados por los partidarios de Lerroux en Barcelona demuestra que son respaldados por una parte significativa del electorado republicano

Cuadro 3.

Candidatos ganadores en las elecciones provinciales de marzo de 1907

\begin{tabular}{|c|c|c|c|c|c|c|c|}
\hline Distritos & \multicolumn{2}{|l|}{ Sc } & PL & \multicolumn{2}{|c|}{ PC } & \multicolumn{2}{|c|}{ RAL* } \\
\hline Barcelona distrito 2 & $\begin{array}{l}\text { D. Millán } \\
\text { B. Maria } \\
\text { R. Albó } \\
\text { S, Gubern }\end{array}$ & $\begin{array}{l}18.476 \\
17.326 \\
17.252 \\
17.038 \\
\end{array}$ & & & & & \\
\hline Barcelona distrito 3 & $\begin{array}{l}\text { A. Nogués } \\
\text { B. Tona } \\
\text { LI. Argemí }\end{array}$ & $\begin{array}{l}9.055 \\
8.898 \\
8.951\end{array}$ & & & & J. Pich** & 7.553 \\
\hline Igualada-Vilafranca *** & $\begin{array}{l}\text { F. Ràfols } \\
\text { S. Coderch } \\
\text { J. Serra }\end{array}$ & $\begin{array}{l}7.169 \\
6.299 \\
5.760 \\
\end{array}$ & & & & & \\
\hline Vilanova-Sant Feliu & $\begin{array}{l}\text { J. Roig } \\
\text { M. Roca } \\
\text { E. Micó }\end{array}$ & $\begin{array}{l}6.146 \\
6.059 \\
5.678\end{array}$ & J. Sostres $(\mathrm{PL})^{* *} 4.160$ & & & & \\
\hline Girona & $\begin{array}{l}\text { J. Ribot } \\
\text { J.M. Vilahur } \\
\text { S. Oliver }\end{array}$ & $\begin{array}{l}7.078 \\
6.923 \\
6.640\end{array}$ & N. Rigau $(P L)^{\star *} 3523$ & & & & \\
\hline Olot-Puigcerdà & $\begin{array}{l}\text { J. Solà-Morales } \\
\text { T. Montagut } \\
\text { I. Riu ** }\end{array}$ & $\begin{array}{l}7.431 \\
7.296 \\
7.038\end{array}$ & & J. Puig (PC) & 8.287 & & \\
\hline
\end{tabular}

$\left(^{*}\right)$ : Republicanos antisolidarios de Lerroux.

$\left({ }^{* *}\right)$ : por la minoría.

$\left.{ }^{* * *}\right)$ : por la minoría sale Torres, con 5.327 . No se ha identificado su nombre y adscripción política.

Fuente: elaboración propia a partir de Camps (1970: 70-74). 
Cuadro 4.

Elecciones provinciales de marzo de 1907

Votos de SC y los republicanos antisolidarios de Lerroux en los distritos de Barcelona

\begin{tabular}{|l|ll|cc|}
\hline \multicolumn{1}{|c|}{ Distritos } & \multicolumn{2}{|c|}{ SC } & \multicolumn{2}{c|}{ RAL* $^{*}$} \\
\hline Barcelona distrito 2 & D. Millán & 18.476 & S. Casals & 11.147 \\
& B. Maria & 17.326 & R. Torras & 11.090 \\
& R. Albó & 17.252 & A. Ulled & 11.084 \\
& S, Gubern & 17.038 & & \\
\hline Barcelona distrito 3 & A. Nogués & 9.055 & J. Pich & 7.553 \\
& B. Tona & 8.898 & P. Villanueva & 7.492 \\
& Ll. Argemí & 8.951 & J. A. Mir & 7.490 \\
\hline
\end{tabular}

Fuente: elaboración propia a partir de Camps (1970: 70-74).

de la ciudad. Un rendimiento que los reafirma en su orientación estratégica. Se debe continuar el camino emprendido en Barcelona desde principios de siglo. Por medio de una intensa labor organizativa, forjadora de un partido de masas, y la confrontación política con los liberales, conservadores y regionalistas, la UR ha logrado su implantación asociativa y electoral en amplios sectores obreros de la ciudad. La alianza electoral con carlistas y regionalistas, aunque pueda reportar más escaños, supone una amenaza para el desarrollo de esta estrategia (Cortina 1992, 1998; Romero 1989: cap. 5; Álvarez Junco 1990: cap. 8; Culla 1986).

\section{Conclusiones}

En este trabajo se ha analizado por qué carlistas, regionalistas y republicanos se coaligan en las elecciones generales de 1907 y pueden conseguir más escaños que concurriendo en solitario. Tienen preferencias derivadas similares _ rechazan la Ley de Jurisdicciones y la manipulación electoral y se manifiestan a favor de la descentralización-y elevadas tasas de descuento: le dan prioridad a los resultados electorales. El sistema electoral de la Restauración es de mayoría relativa, con 34 distritos uninominales y dos multinominales en Cataluña. El PL y el PC, en un escenario de elecciones competitivas, deciden presentar un único candidato en los 32 distritos uninominales donde SC es fuerte; en los otros dos compiten candidatos de ambos partidos. En las elecciones provinciales de marzo de 1907 SC alcanza 19 representantes en los seis distritos donde se presenta, frente a dos del PL, uno del PC y uno de los republicanos antisolidarios de Lerroux.

Las elites carlistas, regionalistas y republicanas conocen estas condiciones institucionales y políticas favorables a la coordinación. De hecho, el cálculo estratégico que les lleva a plantearse la alianza electoral se basa en ellas. Por un lado, saben que en los distritos uninominales resulta ventajoso concentrar el voto en un único candidato. Por otro, están al tanto de la estrategia de coordinación electoral practicada por liberales y conservadores en circunscripciones uninominales disputadas. Además, la experiencia de las elecciones provinciales de marzo les ha suministrado información sobre la ventaja de presentarse juntos. El acuerdo electoral, a pesar de la distancia ideológica entre los partidos coaligados, cuenta con el apoyo del electorado.

Los resultados obtenidos en la investigación respaldan la validez de las preferencias derivadas similares para explicar las alianzas electorales entre partidos alejados en el espectro ideológico. Dada la vocación universal de este mecanismo causal, tendría que funcionar en las restantes coaliciones electorales de esta naturaleza. Para ello se deberían analizar sistemáticamente estos acuerdos electorales en el tiempo —en todas las olas de democratización y contraolas - y en el espacio - las diferentes regiones del mundo-. Por ejemplo, se podría abordar su estudio en Europa y América Latina durante la primera ola y su posterior contraola.

Sin embargo, el análisis empírico desarrollado también muestra la posibilidad de que las preferencias ideológicas antagónicas hagan imposible una alianza electoral entre partidos con preferencias derivadas similares. Lerroux y sus partidarios rechazan el pacto electoral con carlistas y regionalistas y prefieren presentarse en solitario frente a SC, pese a compartir con estas formaciones políticas el rechazo a la Ley de Jurisdicciones y el fraude electoral, incluso corriendo el riesgo de no obtener ningún escaño en el Congreso de los Diputados. De ahí que la relación causal entre las preferencias ideológicas y las preferencias derivadas debería incorporarse a la investigación sobre coordinación electoral.

\section{Agradecimientos}

Agradezco los comentarios y sugerencias del grupo de investigación HistoriaAgraria de Galicia (HISTAGRA) de la Universidad de Santiago de Compostela y de Lourenzo Fernández Prieto, Ignacio Lago, Ramón Máiz y dos evaluadores anónimos de la Revista Internacional de Sociología. Obviamente, la responsabilidad sobre el contenido del texto es enteramente mía. 


\section{NOTAS}

1. Proporcionalidad: "Grado de coincidencia entre proporción de votos obtenidos y proporción de escaños recibidos por un partido en una elección" (Montero, Colomer y Lago, 2005: 357).

2. "Competición electoral en un distrito uninominal en la que los dos o más partidos o candidatos que se disputan el segundo lugar están tan igualados que sus seguidores no son capaces de decidir cuál de ellos será abandonado estratégicamente. Por tanto, los electores votan sinceramente, de modo que compiten más de dos partidos o candidatos" (Montero, Colomer y Lago, 2005: 352).

\section{Referencias Bibliográficas}

Álvarez Junco, J. 1990. El emperador del Paralelo. Lerroux y la demagogia populista. Madrid: Alianza Editorial.

Artola, M. 1974. Partidos y programas políticos. 1808-1936. Madrid: Aguilar.

Balcells, A., J. B. Culla y C. Mir. 1982. Les eleccions generals a Catalunya de 1901 a 1923. Relació dels resultats electorals de 1869 a 1899. Barcelona: Fundació Jaume Bofill.

Bandyopadhyay, S., K. Chatterjee y T. Sjöström. 2011. "Preelectoral Coalitions and Post-election Bargaining". Quarterly Journal of Political Science, 6: 1-53. https:// doi.org/10.1561/100.00010043

Benoit, K. 2004. "Models of Electoral System Change". Electoral Studies 23: 363-389. https://doi.org/10.1016/ S0261-3794(03)00020-9

Blais, A. e I. Indridason. 2007. "Making Candidates Count: The Logic of Electoral Alliances in Two-Round Legislative Elections". The Journal of Politics 69: 193-205. https:// doi.org/10.1111/j.1468-2508.2007.00504.x

Camps, J. de. 1970. Història de la Solidaritat Catalana. Barcelona: Ediciones Destino.

Canal, J. 1998. El carlisme català dins I'Espanya de la Restauració. Un assaig de modernització política (1888-1900). Vic: Eumo Editorial.

Canal, J. 2006. Banderas blancas, boinas rojas: una historia política del carlismo, 1876-1939. Madrid: Marcial Pons.

Colomer, J. M. 2004. "Spain: from Civil War to Proportional Representation". Pp. 253-264 en Handbook of Electoral System Choice, editado por J. M. Colomer. New York: Palgrave Macmillan.

Cortina, M. S. 1992. "Solidaridad Catalana y el republicanismo histórico". Pp. 115-126 en Catalunya i la Restauració. Manresa: Centre d'Estudis del Bages.

Cortina, M. S. 1998. "La Restauració a la Catalunya urbana (1900-1923)". Pp. 121-130 en Actituds polítiques $i$ control social a la Catalunya de la Restauració (18751923), editado por C. Mir. Lleida: Publicacions de I'Estudi General de Lleida.

Cox, G. 1997. Making Votes Count. Strategic Coordination in the World's Electoral Systems. Cambridge: Cambridge University Press.

Cox, G. 2000. "Coordination Electoral". Pp. 49-50 en International Encyclopedia of Elections, editado por R. Rose. London: MacMillan.

Cuadrado, M. 1969. Elecciones y partidos políticos en España. Madrid: Taurus.

Culla, J. B. 1986. El Republicanisme Lerrouxista a Catalunya: 1901-1923. Barcelona: Curial.

Duarte, A. 2004. Història del republicanisme a Catalunya. Lleida: Eumo Editorial.
3. La Ley Provincial de 1882 establece que las diputaciones provinciales se deben renovar mediante elecciones bienales que, por turnos previamente establecidos, se realizan en la mitad de los distritos electorales. Por norma, en cada distrito electoral se elige a cuatro diputados provinciales. El voto es limitado: la proporción entre los votos de cada votante y los escaños es de 3/4 (Peña 1998).

Duverger, M. 1987. Los partidos políticos. México: Fondo de Cultura Económica.

Gerring, J. 2007. Case Study Research: Principles and Practices. Cambridge University Press.

Golder, S. N. 2006. The Logic of Pre-electoral Coalition Formation. Ohio: The Ohio University Press.

Lago, I. 2004. "La coordinación electoral del nacionalismo gallego". Revista Internacional de Sociología 39: 33-61. https://doi.org/10.3989/ris.2004.i39.248

Luebbert, G. M. 1991. Liberalism, Fascism or Social Democracy: Social Classes and the Political Origins of Regimes in Interwar Europe. New York: Oxford University Press.

Martínez, F. 2006a. "Nicolás Salmerón y Alonso. Entre la revolución y la política". Pp. 127-160 en Progresistas. Biografías de reformistas españoles, editado por $\mathrm{J}$. Moreno. Madrid: Taurus.

Martínez, F. 2006b. "La 'redención' por el sufragio. La apuesta política de Nicolás Salmerón y Alonso en el cambio de siglo (1890-1903)". Pp. 61-87 en República y modernidad. El republicanismo en los umbrales del siglo XX, editado por M. Morales. Málaga: Diputación de Málaga.

Martínez, F. 2008a. Nicolás Salmerón y Alonso. Discursos y escritos políticos. Almería: Universidad de Almería.

Martínez, F. 2008b. "Salmerón y las solidaridades". Pp. 136-137 en Solidaritat Catalana i Espanya (19051909), editado por G. Rubí y F. Espinet. Barcelona: Editorial Base.

Molas, I. 1972. Lliga Catalana. Un estudi d'Estasiologia. Barcelona: Edicions 62.

Montero, R., J. M. Colomer e I. Lago. 2005. "Glosario básico de sistemas electorales". Zona Abierta 110-111: 349-358.

Pabón, J. 1952. Cambó. Barcelona: Alpha.

Peña, M. A. 1998. Clientelismo político y poderes periféricos durante la Restauración (1874-1923). Huelva: Universidad de Huelva.

Reid, G. y H. Chapman. 1995. The Social Construction of Democracy, 1870-1990. Houndmills: Macmillan.

Riquer, B. de. 1972. "Les eleccions de la Solidaritat Catalana a Barcelona", Recerques, 2: 93-140.

Riquer, B. de. 1977. Lliga Regionalista: la burgesia catalana i el nacionalisme (1898-1904). Barcelona: Ediciones 62.

Romero, J. 1989. "La rosa de fuego". El obrerismo barcelonés de 1899 a 1909. Madrid: Alianza Editorial.

Rubí, G. y J. Armengol. 2001. "Cataluña”. Pp. 237-282 en El poder de la influencia. Geografía del caciquismo en 
España (1875-1923), dirigido por J. Varela. Madrid: Marcial Pons.

Rubí, G. y J. Armengol. 2012. Vots, electors i corrupció. Una reflexió sobre l'apatia a Ctalunya (1869-1923). Barcelona: Publicacions de l'Abadía de Montserrat.
Sartori, G. 1994. Ingeniería constitucional comparada. Una investigación de las estructuras, incentivos y resultados. México: Fondo de Cultura Económica.

Tsebelis, G. 1990. Nested Games, Rational Choice in Comparative Politics. Berkeley: University of California Press.

\section{Anexo}

\section{Cuadro 1A.}

Distritos multinominales

\begin{tabular}{|c|c|c|c|c|c|c|c|c|c|c|c|c|c|c|c|}
\hline & SC & & & RA & & & PC & & & PL & & & Otros & & \\
\hline Distritos & Votos & $\% V^{* *}$ & $E^{* * *}$ & Votos & $\% \mathrm{~V}$ & $E$ & Votos & $\% \mathrm{~V}$ & $E$ & Votos & $\% \mathrm{~V}$ & $E$ & Votos & $\% \mathrm{~V}$ & $E$ \\
\hline Barcelona & $\begin{array}{|lc|}\text { N. Salmerón } 39.935 \\
\text { F. Cambó } & 39.458 \\
\text { J. Puig } & 39.454 \\
\text { E. Junoy } & 38.996 \\
\text { F. Macià } & 37.113 \\
\text { J.M. Vallès } & 36.501 \\
\text { l. Sunyol } & 35.110\end{array}$ & 70,84 & 7 & \begin{tabular}{|ll} 
J. Sol & 22.081 \\
A. Lerroux & 22.024 \\
N. Estébanez & 21.974 \\
J.M. Serraclara & 21.918 \\
J.A. Mir & 21.699
\end{tabular} & 29,15 & 0 & & & & & & & & & \\
\hline \begin{tabular}{|l|} 
Tarragona- \\
Reus-Falset
\end{tabular} & \begin{tabular}{|lr} 
J. Nogués & 8.994 \\
R. Mayner & 8.087 \\
J. Suelves & 7.096 \\
\end{tabular} & $\begin{array}{l}21,52 \\
19,35 \\
16,98\end{array}$ & 3 & $\begin{array}{l}\text { A. Lerroux } \\
\text { E. Iglesias }\end{array}$ & $\begin{array}{l}1,62 \\
0,16\end{array}$ & 0 & R. Morenes 6.743 & 16,13 & 0 & J. Canellas 5.946 & 14,22 & 0 & 97 & 0,22 & 0 \\
\hline
\end{tabular}

${ }^{*}$ ): Republicanos antisolidarios de Lerroux.

$\left({ }^{* *}\right)$ : 'Porcentaje de votos.

$\left({ }^{* * *}\right)$ : Escaños.

Fuente: elaboración propia a partir de Balcells et al. (1982: 520, 526). 


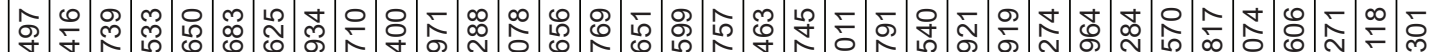

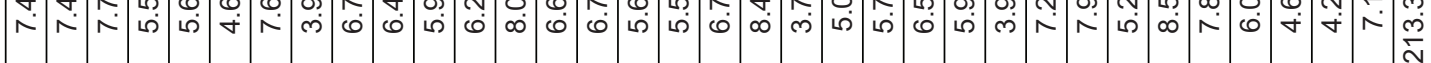

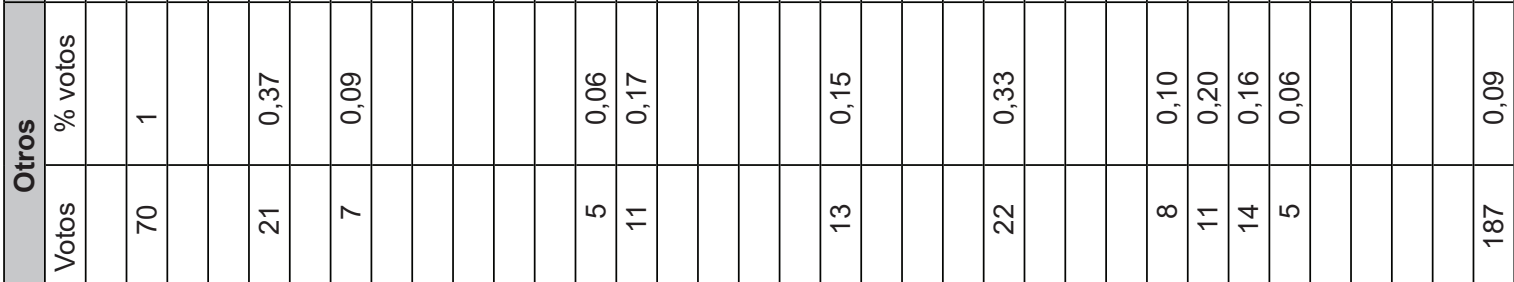

满

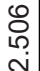

哭

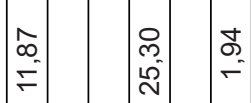

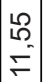

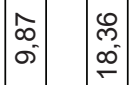

$\underset{\substack{f \\ \hdashline}}{ }$

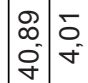

*

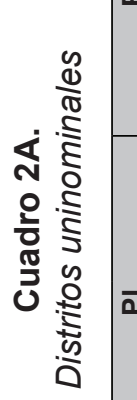

윰

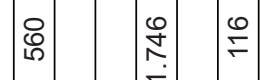

文

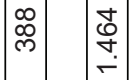

㖉

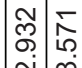

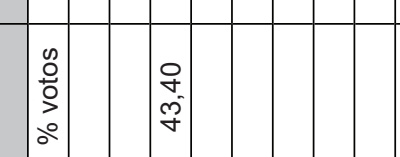

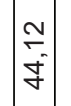

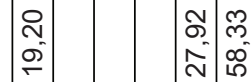

$\frac{\infty}{6}$

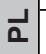

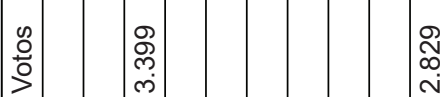

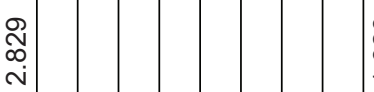

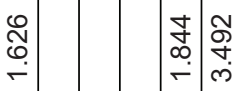

$\stackrel{\circ}{\stackrel{2}{-}}$

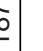

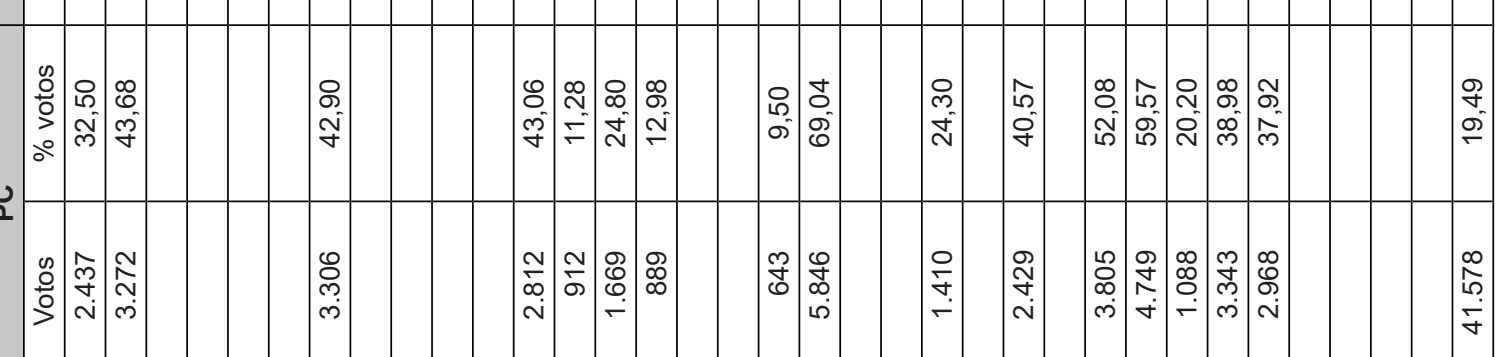

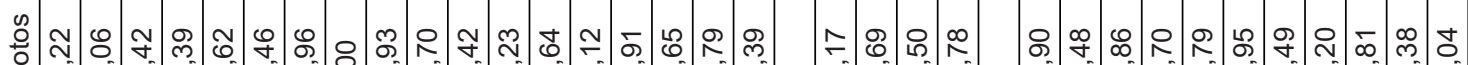

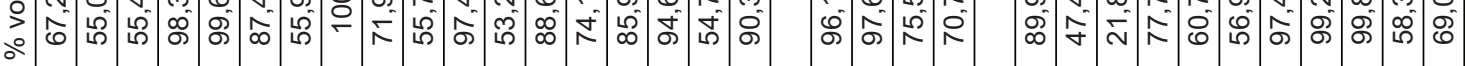

ن)

:

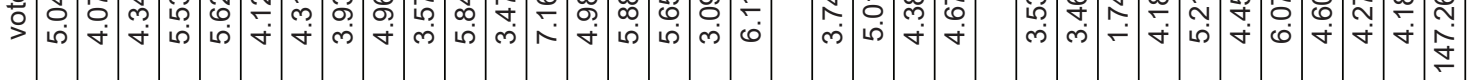




\section{Cuadro 3A.}

Elecciones generales de abril de 1907 Distribución proyectada de votos y votantes

\begin{tabular}{|c|c|}
\hline Distrito & Candidatura \\
\hline | y || & $\begin{array}{l}\text { E. Junoy } \\
\text { F. Macià } \\
\text { J. Puig i Cadafalch } \\
\text { I. Sunyol } \\
\text { J.M. Vallès }\end{array}$ \\
\hline III & $\begin{array}{l}\text { F. Cambó } \\
\text { E. Junoy } \\
\text { F. Macià } \\
\text { I Sunyol } \\
\text { J.M. Vallès }\end{array}$ \\
\hline IV & $\begin{array}{l}\text { F. Cambó } \\
\text { F. Macià } \\
\text { N. Salmerón } \\
\text { I. Sunyol } \\
\text { J.M. Vallès }\end{array}$ \\
\hline V & $\begin{array}{l}\text { F. Macià } \\
\text { J. Puig i Cadafalch } \\
\text { N. Salmerón } \\
\text { I Sunyol } \\
\text { J.M. Vallès }\end{array}$ \\
\hline VI y parte del IX y el X & $\begin{array}{l}\text { F. Cambó } \\
\text { E. Junoy } \\
\text { J. Puig i Cadafalch } \\
\text { N. Salmerón } \\
\text { F. Macià }\end{array}$ \\
\hline VII y parte del IX y el X & $\begin{array}{l}\text { F. Cambó } \\
\text { E. Junoy } \\
\text { J. Puig i Cadafalch } \\
\text { N. Salmerón } \\
\text { J.M. Vallès }\end{array}$ \\
\hline VIII & $\begin{array}{l}\text { F. Cambó } \\
\text { E. Junoy } \\
\text { J. Puig i Cadafalch } \\
\text { N. Salmerón } \\
\text { I. Sunyol }\end{array}$ \\
\hline
\end{tabular}

Fuente: elaboración propia a partir de Camps (1970).

PEDRO LAGO PEÑAS es doctor en Ciencia Política por la Universidad de Santiago de Compostela. Su principal área de investigación es la acción colectiva y los partidos y movimientos sociales. Sus publicaciones han aparecido en Historia y Política o Revista de Estudios Políticos. 